\title{
Magnetic Susceptibility Study of the Heavy Rare Earth Stannate Pyrochlores
}

\author{
V. Bondah-Jagalu ${ }^{1}$ and S. T. Bramwell ${ }^{2}$
}

${ }^{1}$ Department of Chemistry, The University of Edinburgh, The King's Buildings, West Mains Road, Edinburgh, EH9 3JJ, United Kingdom

2 Department of Chemistry, University College London, 20 Gordon Street, London, WC1H 0AJ, United Kingdom

Corresponding authors: e-mail: vin@ed.ac.uk and s.t.bramwell@ucl.ac.uk

\begin{abstract}
The series of magnetic rare earth pyrochlore stannates $\mathrm{R}_{2} \mathrm{Sn}_{2} \mathrm{O}_{7}(\mathrm{R}=$ rare earth, except Ce and Pm) have been investigated by powder susceptibility measurements down to $\mathrm{T}=1.8 \mathrm{~K}$. The results are compared to results for the analogous titanate series, which are well-known frustrated magnets. Unlike the titanates, the whole series can be formed in the cubic pyrochlore structure. Possible experimental advantages of studying the stannates are discussed.
\end{abstract}

\section{Introduction}

The magnetic properties of the pyrochlore compounds can be very unusual on account of the high degree of frustration between antiferromagnetically coupled magnetic ions [1]. Cubic pyrochlore oxides $\mathrm{A}_{2} \mathrm{~B}_{2} \mathrm{O}_{6} \mathrm{O}^{\prime}$ are described by the face centred space group $F d-3 m$ where the trivalent $\mathrm{A}$ atoms are situated on the $16 \mathrm{~d}$ sites, the tetravalent $\mathrm{B}$ atoms are on the $16 \mathrm{c}$ sites and the oxygen atoms are in the $48 \mathrm{f}(\mathrm{O})$ and $8 \mathrm{~b}\left(\mathrm{O}^{\prime}\right)$ sites. The $\mathrm{A}$ and the $\mathrm{B}$ atoms form identical interpenetrating sublattices that are spatially displaced from each other by $\left\langle\frac{1}{2}, \frac{1}{2}, \frac{1}{2}\right\rangle$. Each lattice can be described as a three dimensional array of corner linked tetrahedra and it is this basic tetrahedral motif that gives rise to the geometrical frustration of antiferromagnetic interactions. The A site is coordinated by two oxygen atoms $\left(\mathrm{O}^{\prime}\right)$ at the centre of the tetrahedra and by six oxygen atoms arranged in a puckered hexagonal ring around the metal ions. The structural details of pyrochlore compounds are well described in a very comprehensive review of the oxide pyrochlores [2]. The tetrahedral lattice is a feature of many other materials, examples including ternary fluorides (e.g. $\left.\mathrm{NH}_{4} \mathrm{CoAlF}_{6} \sqrt[3 \|]{3}\right)$ and spinels (e.g. $\mathrm{MgCr}_{2} \mathrm{O}_{4}$ [4]).

Investigation of the magnetic properties of the pyrochlores has been pioneered mainly by J .E. Greedan and collaborators. Of particular interest is the discovery [5] that several compounds of the form $\mathrm{R}_{2} \mathrm{Mo}_{2} \mathrm{O}_{7}(\mathrm{R}=$ rare earth or $\mathrm{Y})$ show frozen in magnetic disorder in the apparent absence of chemical disorder. For these compounds the molybdenum(IV) ions are magnetic $\left(\mathrm{d}^{2}, \mathrm{~S}=1\right)$ and the $\mathrm{R}$ ion can be chosen to be nonmagnetic. Depending on the rare earth, the molybdates may be metallic $(\mathrm{R}=\mathrm{Sm}, \mathrm{Nd}, \mathrm{Gd})$ or semiconducting $(\mathrm{R}=\mathrm{Y}, \mathrm{Tb}-\mathrm{Yb})$ [6, [] and it is not entirely clear to what extend local moment models of frustration would apply to these materials. It therefore remains of interest to characterise new families of magnetic pyrochlore materials with localised moments. Here we describe a susceptibility study of a family of rare earth stannates $\mathrm{R}_{2} \mathrm{Sn}_{2} \mathrm{O}_{7}$ in which the $\mathrm{Sn}(\mathrm{IV})$ ion is nonmagnetic and the rare-earth ions are chosen to be magnetic. This study follows on from an investigation of the isostructural titanate materials $\mathrm{R}_{2} \mathrm{Ti}_{2} \mathrm{O}_{7}$ [8] that in turn developed the early work of Cashion [9] and Blöte [10]. 
While investigating $\mathrm{Ho}_{2} \mathrm{Ti}_{2} \mathrm{O}_{7}$ it was realised [11, 12] that in the presence of local $\langle 111\rangle$ easy axis anisotropy, frustration arose from a net ferromagnetic coupling between spins which has subsequently been established to be largely dipolar in origin [13]. The simple $\langle 111\rangle$ Ising ferromagnet was termed the "spin ice" model on account of its precise mapping onto the problem of proton disorder in ice. It gives a good first approximation to the properties of $\mathrm{Ho}_{2} \mathrm{Ti}_{2} \mathrm{O}_{7}$ [9] and $\mathrm{Dy}_{2} \mathrm{Ti}_{2} \mathrm{O}_{7}$ [14. Following on from the titanates, we decided to look at the magnetic properties of the rare earth stannates, whose structural properties have been well studied [15, 16, 17]. The compounds are cubic, with a linear relationship between the radius of the lanthanide and the lattice. Non-cubic pyrochlore stannates do exist, such as $\mathrm{Bi}_{2} \mathrm{Sn}_{2} \mathrm{O}_{7}$ [18, 19].

\section{Experimental}

Our samples were prepared from $\mathrm{SnO}_{2}$ and $\mathrm{R}_{2} \mathrm{O}_{3}$ $(\mathrm{R}=\mathrm{La}, \mathrm{Nd}, \mathrm{Sm}, \mathrm{Eu}, \mathrm{Gd}, \mathrm{Dy}, \mathrm{Ho}, \mathrm{Er}, \mathrm{Tm}, \mathrm{Yb}$, Lu and Y) ordered from Strem and Aldrich, all of a minimum of $99.9 \%$ purity. For the praseodymium and terbium stannate, $\mathrm{Pr}_{6} \mathrm{O}_{11}$ and $\mathrm{Tb}_{4} \mathrm{O}_{7}$ were used respectively and it was assumed that the excess oxygen would escape as a gas during annealing. It was not possible to prepare cerium stannate in this way, since the cerium remains as $\mathrm{Ce}^{+4}$. However, because of this fact, it would be possible to make $\mathrm{La}_{2} \mathrm{Ce}_{2} \mathrm{O}_{7}$ [20], although this material adopts a disordered fluorite structure. The oxides were ground, pressed into pellets and heated in air to $1400^{\circ} \mathrm{C}$ for 4 hours, this length of time being found sufficient for all of the compounds. The X-ray diffraction refinement of room temperature data gave lattice constants consistent with the literature values [17.

Magnetisation was measured in the range $1.8 \mathrm{~K}$ to $300 \mathrm{~K}$ at an applied field of $10 \mathrm{Oe}(T<20 \mathrm{~K})$ and 100 Oe $(\mathrm{T}<20 \mathrm{~K})$ using a Quantum Design SQUID magnetometer, and divided by field and amount of substance to give the susceptibility per mole of lanthanide. The low field susceptibility was scaled to fit the higher field data, as the field positioning at 10 Oe is not very accurate. Zero- field cooled versus field cooled susceptibility was determined in an applied field of 10 Oe between $\mathrm{T}=1.84 \mathrm{~K}$ and $\mathrm{T}=$ $20 \mathrm{~K}$, and magnetisation versus field isotherms were measured at $\mathrm{T}=1.8 \mathrm{~K}$ in applied fields of up to $7 \mathrm{~T}$. Estimated Curie-Weiss temperatures and magnetic moments are listed in table 1 . Individual curves of effective moment $\mu_{e f f}=\sqrt{8 \chi T}$ versus temperature are shown in figure 1.

\begin{tabular}{|l|c|c|c|c|c|c|c|c|}
\hline & & $\begin{array}{c}\text { Van } \\
\text { Vleck }\end{array}$ & $\begin{array}{c}\theta \\
K\end{array}$ & error & $\begin{array}{c}\mathrm{C} \\
\text { eerg mol }\end{array}{ }^{-1} \mathrm{~K}$ & error & $\begin{array}{c}\mu \\
/ \mu_{\mathrm{B}} \text { atom }\end{array}$ & error \\
\hline $\mathrm{Pr}_{2} \mathrm{Sn}_{2} \mathrm{O}_{7}$ & $5-15 \mathrm{~K}$ & 0 & -0.03 & 0.02 & 1.121 & 0.002 & 2.99 & 0.01 \\
\hline $\mathrm{Nd}_{2} \mathrm{Sn}_{2} \mathrm{O}_{7}$ & $5-15 \mathrm{~K}$ & 0 & -0.32 & 0.02 & 0.924 & 0.002 & 2.72 & 0.01 \\
\hline $\mathrm{Sm}_{2} \mathrm{Sn}_{2} \mathrm{O}_{7}$ & $5-20 \mathrm{~K}$ & 0.00143 & 1.36 & 0.21 & 0.012 & 0.000 & 0.30 & 0.01 \\
\hline $\mathrm{Eu}_{2} \mathrm{Sn}_{2} \mathrm{O}_{7}$ & & 0.008 & & & & & & \\
\hline $\mathrm{Gd}_{2} \mathrm{Sn}_{2} \mathrm{O}_{7}$ & $50-100 \mathrm{~K}$ & 0 & -9.63 & 0.12 & 8.119 & 0.013 & 8.06 & 0.02 \\
\hline $\mathrm{Tb}_{2} \mathrm{Sn}_{2} \mathrm{O}_{7}$ & $50-100 \mathrm{~K}$ & 0 & -11.05 & 0.16 & 12.189 & 0.025 & 9.87 & 0.03 \\
\hline $\mathrm{Dy}_{2} \mathrm{Sn}_{2} \mathrm{O}_{7}$ & $2-10 \mathrm{~K}$ & 0 & -0.54 & 0.03 & 13.764 & 0.068 & 10.49 & 0.07 \\
\hline $\mathrm{H}_{2} \mathrm{Sn}_{2} \mathrm{O}_{7}$ & $2-10 \mathrm{~K}$ & 0 & -0.69 & 0.03 & 14.365 & 0.072 & 10.72 & 0.08 \\
\hline $\mathrm{Er}_{2} \mathrm{Sn}_{2} \mathrm{O}_{7}$ & $50-100 \mathrm{~K}$ & 0 & -18.03 & 0.18 & 11.808 & 0.027 & 9.72 & 0.03 \\
\hline $\mathrm{Tm}_{2} \mathrm{Sn}_{2} \mathrm{O}_{7}$ & & 0.09 & & & & & & \\
\hline $\mathrm{Yb}_{2} \mathrm{Sn}_{2} \mathrm{O}_{7}$ & $2-10 \mathrm{~K}$ & 0 & 0.43 & 0.01 & 1.384 & 0.002 & 3.33 & 0.01 \\
\hline
\end{tabular}

Table 1: The Curie-Weiss temperature, constant and magnetic moment for the rare earth pyrochlore stannates, estimated by extrapolating data from the given temperature ranges and "if required" applying the Van Vleck paramagnetism correction.

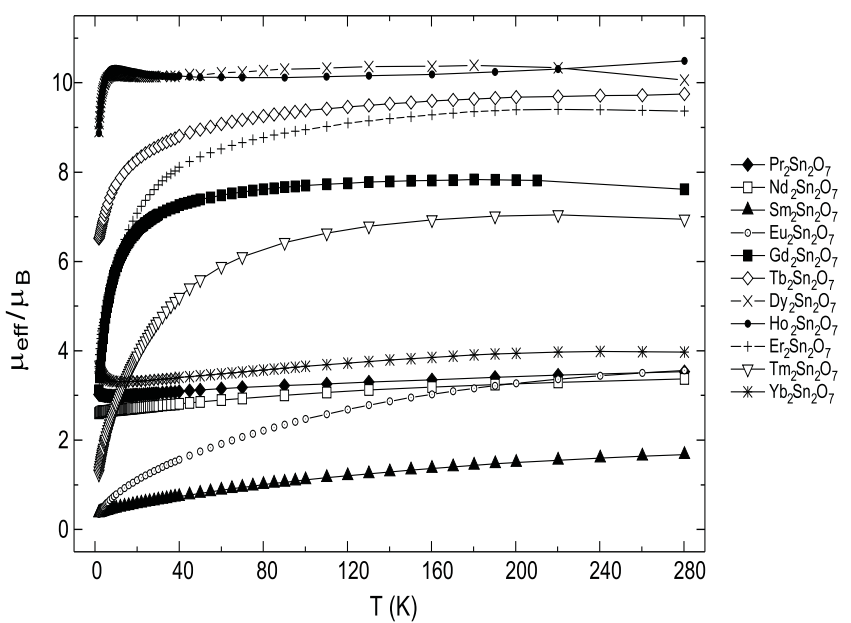

Figure 1 : The effective moments $\mu_{\text {eff }}$ versus temperature $T$ for the rare earth pyrochlore stannates. The moments are derived from measured molar susceptibility $\chi$ (in cgs units) via the relation $\mu_{\text {eff }}=\sqrt{8 \chi T}$.

\section{Results and Discussion}

$\mathrm{R}_{2} \mathrm{Sn}_{2} \mathrm{O}_{7}(\mathrm{R}=\mathrm{Nd}, \mathrm{Tb}, \mathrm{Dy}, \mathrm{Ho}, \mathrm{Er}, \mathrm{Yb})$ almost certainly have doublet ground states with strong single ion anisotropy. The latter is evidenced by the fact that the magnetisation approaches saturation at 
roughly half the free ion value. Amongst this group all except $\mathrm{Yb}_{2} \mathrm{Sn}_{2} \mathrm{O}_{7}$ have Curie constants that are consistent with a ground state for $R^{3+}$ consisting mainly of $M_{J}= \pm J$ for the free ion ground term. For $\mathrm{R}=\mathrm{Nd}$, Gd, Dy, Ho, Er, Tm, Yb the magnetic behaviour is extremely close to that of the analogous titanate. Thus one expects "dipolar spin ice" behaviour for $\mathrm{R}=\mathrm{Ho}$, Dy and ordering at $\mathrm{T}<1.8$ $\mathrm{K}$ for $\mathrm{R}=\mathrm{Nd}, \mathrm{Er}, \mathrm{Yb}$, while $\mathrm{R}=$ Tm clearly has a singlet ground state derived from crystal field splitting of the free ion term [13, 10, 21]. Both $\mathrm{Er}_{2} \mathrm{Sn}_{2} \mathrm{O}_{7}$ and $\mathrm{Er}_{2} \mathrm{Ti}_{2} \mathrm{O}_{7}$ exhibit a pronounced FC-ZFC splitting in the susceptibility at about $3.4 \mathrm{~K}$. This feature needs to be investigated further. For $\mathrm{Tb}_{2} \mathrm{Sn}_{2} \mathrm{O}_{7}$, the Curie-Weiss temperature $\theta_{C W}=-11.1 \mathrm{~K}$, is apparently much less than in $\mathrm{Tb}_{2} \mathrm{Ti}_{2} \mathrm{O}_{7}, \theta_{C W}=-19 \mathrm{~K}$ [22]. It is possible that our sample of $\mathrm{Tb}_{2} \mathrm{Sn}_{2} \mathrm{O}_{7}$ is slightly non-stoichiometric. Consistent with this, we observe a slight FC-ZFC splitting in the susceptibility below $4.5 \mathrm{~K}$, which is not reported for the titanate. The latter has been described as a cooperative paramagnet with no sign of magnetic order down to $\mathrm{T}=0.07 \mathrm{~K} 23$.

There have not been detailed reports of the magnetism of titanates analogous to $\mathrm{R}_{2} \mathrm{Sn}_{2} \mathrm{O}_{7}$ with $\mathrm{R}=$ $\mathrm{Pr}, \mathrm{Sm}, \mathrm{Eu}$, so we consider their behaviour in more detail.

$\mathrm{Pr}_{2} \mathrm{Sn}_{2} \mathrm{O}_{7}$ : The saturation magnetisation and effective moment approach the free ion value, while the Curie-Weiss constant is small. This suggests that crystal field effects and magnetic coupling are small in this material. This behaviour can be contrasted with $\mathrm{Pr}_{2} \mathrm{O}_{3}$ 24, 25] which has a large $\theta_{C W}$ $\approx-60 \mathrm{~K}$ arising from the crystal field splitting of the $\mathrm{H}_{6}$ free ion term of $\mathrm{Pr}^{3+}$ to give a ground state singlet and low lying doublet.

$\mathrm{Sm}_{2} \mathrm{Sn}_{2} \mathrm{O}_{7}$ : The effective moment versus temperature appears to have a term in (temperature $)^{\frac{1}{2}}$ dominating at high temperature, suggestive of a Van Vleck paramagnetism. It is typical for the susceptibility of $\mathrm{Sm}^{3+}$ salts to be dominated by the Van Vleck term near room temperature, and to show increasingly Curie-Weiss behaviour at low temperature [26]. Ascribing the value $0.00143 \mathrm{erg} \mathrm{Oe}^{-2}$ $\mathrm{mol}^{-1}$ to the Van Vleck term gives a Curie-Weiss temperature of $1.36 \mathrm{~K}$ and moment derived from the Curie constant of 0.30 , which is less than the moment of the ${ }^{6} \mathrm{H}_{\frac{5}{2}}$ free ion term, $0.84 \mu_{B}$ atom $^{-1}$. This reduction in moment is consistent with the ground term in $\mathrm{Sm}_{2} \mathrm{Sn}_{2} \mathrm{O}_{7}$ being a doublet dominated by free ion $\mathrm{M}_{J}$ levels with $\mathrm{M}_{J}$ significantly less than the maximum, $\frac{5}{2}$. A similar effect is observed in $\mathrm{Sm}_{2} \mathrm{O}_{3}$, which shows a similar susceptibility curve [26].

$\mathrm{Eu}_{2} \mathrm{Sn}_{2} \mathrm{O}_{7}$ : The susceptibility is roughly constant below $100 \mathrm{~K}$, behaviour typical for $\mathrm{Eu}^{3+}$, in which the free ion ${ }^{7} \mathrm{~F}_{0}$ ground term is non-magnetic, leaving only the Van Vleck term. Similar behaviour is observed in $\mathrm{Eu}_{2} \mathrm{O}_{3}$ [26]. The observed magnitude

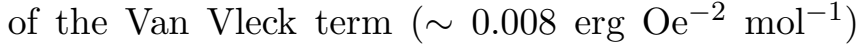
in $\mathrm{Eu}_{2} \mathrm{Sn}_{2} \mathrm{O}_{7}$ is close to that of $\mathrm{Eu}_{2} \mathrm{O}_{3}$, which has been shown to be enhanced by anisotropic exchange between the $\mathrm{Eu}$ ions [27].

\section{Concluding Remarks}

The rare earth stannates can be prepared in the cubic form across the whole lanthanide series and make an ideal system for the comparative study of magnetic properties. Experimental investigation of the series to lower temperatures is in progress. The presence of Sn in the structure will facilitate experimental investigation of these materials by Mossbauer ${ }^{119} \mathrm{Sn}$ spectroscopy. For example, using this technique, $\mathrm{Sn}_{2} \mathrm{~B}_{2} \mathrm{O}_{7}(\mathrm{~B}=\mathrm{Nb}$, Ta) have been shown to be actually of the form $\mathrm{Sn}_{2-2 u}^{2+}\left(\mathrm{Sn}_{2 u}^{4+} \mathrm{B}_{2-u}^{5+}\right) \mathrm{O}_{7-(5 / 2) u}$ ; $u=0.18-0.20$ ) [28]. Solid solutions of the type $\mathrm{R}_{x} \mathrm{R}_{1-x} \mathrm{Sn}_{2} \mathrm{O}_{7}\left(\mathrm{R}=\right.$ rare earth, $\left.\mathrm{R}^{\prime}=\mathrm{Ln}, \mathrm{Y}\right)$ can also be formed. In this context, ${ }^{119} \mathrm{Sn}$ MAS NMR spectroscopy has been shown to be a particularly powerful probe of local cation geometry on account of the very large ${ }^{119} \mathrm{Sn}$ chemical shifts [29]. We finally note that, to complete the series, cerium stannate pyrochlore $\left(\mathrm{Ce}_{2} \mathrm{Sn}_{2} \mathrm{O}_{7}\right)$ has recently been prepared for the first time [30].

\section{References}

[1] P. W. Anderson, Phys. Rev. 102, 1008 (1956).

[2] M. A. Subramanian, G. Aravamudan and G. V. S. Rao, Solid State Chem. 15, 55 (1983).

[3] N. P. Raju et al., Phys. Rev. B 58, 5550 (1998).

[4] H. Shaked, J.M. Hastings and L.M. Corliss, Phys. Rev. B 1, 3116 (1970). 
[5] N. Ali et al., J. Solid State Chem. 83,178 (1989); J. E. Greedan et al., Phys. Rev. B 43, 5682 (1991).

[6] J. E. Greedan, M. Sato, N. Ali and W. R. Datars, J. Solid State Chem. 68, 300 (1987)

[7] Y. Taguchi and Y. Tokura, Phys. Rev. B 60, 10280 (1999).

[8] S. T. Bramwell, M. Field, M. J. Harris and I. P. Parkin, J. Phys. Cond. Matt. 12, 483 (2000).

[9] J. D. Cashion, et al., J. Mater. Sci. 3, 402 (1968).

[10] H. W. J. Blöte, R. F. Wielinga and W. J. Huiskamp, Physica 43, 549 (1969).

[11] M. J. Harris et al., Phys. Rev. Lett. 79, 2554 (1997).

[12] S. T. Bramwell and M. J. Harris, J. Phys.: Condens. Matter 10, L215 (1998).

[13] B. C. Den Hertog and M. J. P. Gingras, Phys. Rev. Lett. 84, 3430 (2000).

[14] A. P. Ramirez et al., Nature 399, 333 (1999).

[15] C. G. Whinfrey, D. W. Eckart and A. Tauber, J. Am. Chem. Soc 82, 2695 (1960); C. G. Whinfrey and A. Tauber, J. Am. Chem. Soc 83, 755 (1961).

[16] F. Brisse and O. Knop, Can. J. Chem. 46, 859 (1968).

[17] B. J. Kennedy, B. A. Hunter and C. J. Howard, J. Solid State Chem. 130, 58 (1997).

[18] R. S. Roth, J. Research Natl. Bur. Standards 56, 17 (1956).

[19] V. Ravi et al., Mater. Lett. 40, 11 (1999).

[20] F. Brisse and O. Knop, Can. J. Chem. 45, 609 (1967).

[21] M. P. Zinkin et al., J. Phys.: Condens. Matter 8, 193 (1996).
[22] J. S. Gardner, B. D. Gaulin and D. McK. Paul, J. Crystal Growth 191, 740 (1998).

[23] J. S. Gardner et al., Phys. Rev. Lett. 82, 1012 (1999).

[24] S. Kern, J. Chem Phys. 40, 208 (1964).

[25] R. C. Vickery and A. Ruben, J. Chem. Soc., 510 (1959)

[26] A. S. Borovik-Romanov and N. M. Kreines, Sov. Phys. JETP 2, 657 (1956).

[27] N. L. Huang and J. H. Van Vleck, J. Appl. Phys. 40, 1144 (1969).

[28] D. J. Stewart et al., Can. J. Chem. 51, 1041 (1973).

[29] C. P. Grey et al., J. Am. Chem. Soc. 111, 505 (1989).

[30] B. Tolla et al., Comptes Rendus Acad. Sci, Series II CHIMIE 2, 139 (1999). 